\title{
Model Risk in Incomplete Markets with Jumps
}

\author{
Nils Detering and Natalie Packham
}

\begin{abstract}
We are concerned with determining the model risk of contingent claims when markets are incomplete. Contrary to existing measures of model risk, typically based on price discrepancies between models, we develop value-at-risk and expected shortfall measures based on realized P\&L from model risk, resp. model risk and some residual market risk. This is motivated, e.g., by financial regulators' plans to introduce extra capital charges for model risk. In an incomplete market setting, we also investigate the question of hedge quality when using hedging strategies from a (deliberately) misspecified model, for example, because the misspecified model is a simplified model where hedges are easily determined. An application to energy markets demonstrates the degree of model error.
\end{abstract}

\section{Introduction}

We are concerned with determining model risk of contingent claims when market models are incomplete. Contrary to existing measures of model risk, based on price discrepancies between models, e.g., [8, 26], we develop measures based on the realized P\&L from model risk. This is motivated by financial regulators' plans to introduce extra capital charges for model risk, e.g., [5, 13, 17]. In a complete and frictionless market model, the "residual" $\mathrm{P} \& \mathrm{~L}$ observed on a perfectly hedged position is due to pricing and hedging in a misspecified model. The distribution of this $\mathrm{P} \& \mathrm{~L}$ can therefore be taken as an input for specifying measures of model risk,

This work was financially supported by the Frankfurt Institute for Risk Management and Regulation (FIRM) and by the Europlace Institute of Finance.

\footnotetext{
N. Detering $(\bowtie)$

Department of Mathematics, University of Munich, Theresienstraße 39,

80333 Munich, Germany

e-mail: n.detering@math.lmu.de

N. Packham

Frankfurt School of Finance \& Management, Sonnemannstr. 9-11,

60314 Frankfurt am Main, Germany

e-mail: n.packham@fs.de

(C) The Author(s) 2015

K. Glau et al. (eds.), Innovations in Quantitative Risk Management,

Springer Proceedings in Mathematics \& Statistics 99,

DOI 10.1007/978-3-319-09114-3_3
} 
such as expected loss, value-at-risk, or expected shortfall, [10]. In an incomplete market, model risk cannot be entirely isolated from market risk by hedging, and further, it is not a priori clear, which hedging strategies are most effective under model uncertainty. The purpose of this paper is to investigate these questions.

The analysis in [10] is primarily focussed on complete and frictionless market models, as this allows for a convenient separation into P\&L from market risk and $\mathrm{P} \& \mathrm{~L}$ from model risk: Since market risk is hedgeable, any remaining $\mathrm{P} \& \mathrm{~L}$ is due to pricing and hedging in a misspecified model. In the setting of incomplete markets, one would rather distinguish between hedgeable and unhedgeable (or residual) $\mathrm{P} \& \mathrm{~L}$, expressing that the unhedgeable $\mathrm{P} \& \mathrm{~L}$ refers to model uncertainty and some unhedged market risk. However, from a practical perspective, as an institution needs to take care of both market risk and model risk-either through hedging or through capital requirements - the distinction is of minor importance.

In addition, the determination and choice of effective hedging strategies in incomplete markets is not as straightforward as the replicating argument in a complete market, but is of high practical relevance. The techniques developed in this paper are suitable to comparing the effectiveness of hedging strategies in incomplete markets under model uncertainty.

Model risk is associated with uncertainty about the model or probability measure that governs the probabilistic behavior of unknown outcomes. In this context, uncertainty refers to uncertainty in the Knightian sense, e.g., [16, 23], in which case the model uncertainty or model ambiguity is expressed by a set of probability measures, each of which defines a valid pricing and hedging model.

A set of axioms for measures of model risk, in the spirit of coherent and convex risk measures [1, 18], was put forward by [8]. A popular measure fulfilling these axioms is a contingent claim's price range across the set of models expressing the model uncertainty. This measure is generalized by [2] to account for a distribution on the model set. It thus allows to incorporate the likelihood of the models into the price range and as such to derive value-at-risk and expected shortfall type measures. However, these measures do not account for the potential losses from model risk realized when hedging in a misspecified model. In a complete market setup, [10] develop value-at-risk and expected shortfall measures on the distribution of losses from model risk, and show that these measures fulfill the axioms for model risk (with the usual exception of value-at-risk not being subadditive).

As a generalization of [10], we develop measures for unhedged risk in incomplete markets, comprising both market and model risk. This applies, for example, when asset price processes are subject to jumps under the pricing measure, where, if at all, perfect replication of contingent claims is possible only under conditions not met in practice (such as infinitely many hedging instruments). Furthermore, in an incomplete market setting, we investigate the question of hedge quality when using hedging strategies from a (deliberately) misspecified model, for example, because the misspecified model is a simplified model where hedges are easily determined. A typical case could be to use a simplified complete market model to determine a replication strategy, when it is known that the actual market is incomplete. 
Several simulation studies investigate the risk from hedging in a simplified model, e.g., $[11,24,25]$. However, to the best of our knowledge, this is never compared to the residual risk in the alternative model when following a risk-minimizing strategy. Yet, this comparison is important for selecting an appropriate model for pricing and hedging.

In a case study, we study the respective loss distributions and measures when applied to options on energy futures. Empirical returns in the energy spot and future markets behave in a spiky way and thus need to be modeled with jump processes. However, to reduce the computational cost and to attain a parsimonious model, often simplified continuous asset price processes are assumed. Based on the measures of model risk, we assess the quality and robustness of hedging in a continuous asset price model when the underlying price process has jumps relative to determining hedges in the jump model itself. As asset price models, we employ continuous and pure-jump versions of the Schwartz model [27], calibrated to the spot market at the Nordic energy exchange Nord Pool.

The paper is structured as follows: In Sect. 2, we construct the loss variable and loss distribution relevant for model risk. Section 3 defines measures on the distribution of losses from model risk and relates them to the axioms for measures of model uncertainty introduced by [8]. In Sect. 4, we introduce a way of measuring the relative losses from hedging in a misspecified model as opposed to hedging in the appropriate model. Finally, Sect. 5 contains a case study from the energy market to illustrate the relative loss measure and draw conclusions about the quality of hedging strategies determined in a complete model with continuous asset price processes, when the underlying market is in fact subject to jumps.

\section{Losses from Hedged Positions}

In this section, we formalize the market setup and the loss process expressing the residual losses from a hedged position. In the case of a complete and frictionless market, these losses correspond to model risk, whereas in the case of an incomplete market, these losses comprise in addition the market risk that is not hedged away.

\subsection{Market and Model Setup}

We begin with a standard market setup under model certainty, as in e.g., [22]. On a probability space $(\Omega, \mathcal{F}, \overline{\mathbb{Q}})$ endowed with a filtration $\left(\mathcal{F}_{t}\right)_{t \geq 0}$ satisfying the "usual hypotheses" are defined adapted asset price processes $\left(S_{t}^{j}\right)_{t \geq 0}, j=0, \ldots, d$. The asset with price process $S^{0}$ represents the money market account, whereas $S^{1}, \ldots, S^{d}$ are risky assets. All prices are discounted, that is, expressed in units of the money 
market account, and $\overline{\mathbb{Q}}$-martingales, with $\overline{\mathbb{Q}}$ a martingale measure equivalent to the objective probability measure.

Throughout we shall assume that $S$ is a Markov process. This applies to many models commonly used in practice, such as the Black-Scholes model, exponential Lévy models, exponential additive models, and stochastic volatility models, such as the Heston model. We shall see below that the Markov assumption simplifies the analysis considerably.

Fixing a time horizon $T$, we consider European-type claims with $\mathcal{F}_{T}$-measurable integrable payoff. Other claims, in particular, path-dependent options, such as Barrier options, can be integrated into the analysis; we refer to [10] for the more general case.

In addition to the risky assets $S=\left(S^{1}, \ldots, S^{d}\right)$, there may be tradeable options maturing at $T$ written on $S$, with observable market prices at time 0 , so-called benchmark instruments. Their $\mathcal{F}_{T}$-measurable payoffs are denoted by $\left(H_{i}\right)_{i \in I}$, and their observed market prices by $C_{i}^{*}, i \in I$, or by $\left[C_{i}^{\text {bid }}, C_{i}^{\text {ask }}\right], i \in I$, if no unique price is available. These benchmark instruments can be used for static hedging, potentially reducing a claim's model risk.

A trading strategy is a predictable process $\Phi=\left(\phi^{0}, \ldots, \phi^{d}, u_{1}, \ldots, u_{I}\right)$, where $\phi^{j}=\left(\phi_{t}^{j}\right)_{t \geq 0}$ denotes the holdings in asset $j$ and $u_{i} \in \mathbb{R}$ denotes the static holding of benchmark instrument $i$. The time- $t$ value of the portfolio is $V_{t}(\Phi)=\sum_{j=0}^{d} \phi_{t}^{j} S_{t}^{j}+$ $\sum_{i=1}^{I} u_{i} H_{t}^{i}$, with $H_{t}^{i}, i=1, \ldots, I$, the time- $t$ prices of the benchmark instruments. To rule out arbitrage opportunities, we require that $\Phi$ is admissible. Further, $\Phi$ is assumed to be self-financing, that is, $\mathrm{d} V_{t}(\Phi)=\sum_{j=1}^{d} \phi_{t}^{j} \mathrm{~d} S_{t}^{j}+\sum_{i=1}^{I} u_{i} \mathrm{~d} H_{t}^{i}, t \geq 0$.

A contingent claim with $\mathcal{F}_{T}$-measurable payoff $X$ is hedgeable if there exists a replicating strategy, i.e., a self-financing trading strategy $\Phi$ such that $V_{T}(\Phi)=X$. Hedging eliminates any P\&L arising from market risk, and, because of the absence of arbitrage opportunities, the claim's price process and the price of the hedging strategy agree for all $0 \leq t \leq T$. In an incomplete market, in the absence of a replicating strategy, losses from market risk may be eliminated or reduced by super-replicating strategies, e.g., [14], or by risk-minimizing strategies, e.g., [19, 20], but some P\&L due to market risk remains.

Aside from market risk, a stakeholder (trader, hedger, shareholder, regulator) may be concerned about model risk when pricing and hedging a contingent claim. Model risk refers to potential losses from mispricing and mishedging, because model $\overline{\mathbb{Q}}$ is possibly misspecified. This uncertainty regarding model $\overline{\mathbb{Q}}$ is captured by a set $\mathcal{Q}$ of martingale measures for the asset price processes, e.g., [8, 9], which may incorporate uncertainty about both the model type and model parameters.

Let

$$
\mathcal{C}=\left\{X \in \sigma\left(S_{T}\right) \mid \sup _{\mathbb{Q} \in \mathcal{Q}} \mathbb{E}\left[X^{2}\right]<\infty\right\},
$$

be the set of contingent claims under consideration, where we require squareintegrability, because for claims with finite second moments quadratic minimizing hedging strategies exist, which will be employed later. The set of trading strategies 
considered is

$$
\begin{gathered}
\mathcal{S}=\left\{\Phi \mid \Phi \text { admissible, predictable, self-financing, } \Phi_{t} \in \sigma\left(S_{t}\right), \forall t \geq 0\right. \\
\text { and } \left.\overline{\mathbb{E}}\left[\int_{0}^{T}\left(\phi^{j}\right)^{2} \mathrm{~d}\left[S^{j}, S^{j}\right]\right]<\infty, j=0, \ldots, d\right\} .
\end{gathered}
$$

The condition $\Phi_{t} \in \sigma\left(S_{t}\right)$ implies that the hedging strategy is a Markov process.

Working on a set of measures requires further conditions, in particular, as the measures in $\mathcal{Q}$ need not be absolutely continuous with respect to $\overline{\mathbb{Q}}$. More specifically, the asset price processes must be consistent under all measures and specifying trading strategies requires the notion of a stochastic integral with respect to the set of measures.

In case the models in $\mathcal{Q}$ are diffusion processes, [28] develop the necessary tools from stochastic analysis, such as existence of a stochastic integral, martingale representation, etc. Although this restricts the joint occurrence of certain probability measures, it does not exclude any particular measure. For our purposes, this limitation does not play a role, as we are primarily interested in choosing a rich set of possible models to cover the model uncertainty. For details, we refer to [10].

In the general case, we pose the following condition on the set of measures $\mathcal{Q}$, which ensures that all objects are well defined when working with uncountably many measures.

Assumption 1 There exists a universal version of the stochastic integral $\int_{0}^{t} \phi \mathrm{d} S$, $\phi \in \mathcal{S}$. In addition, for all $\mathbb{Q} \in \mathcal{Q}$, the integral coincides $\mathbb{Q}$-a.s. with the usual probabilistic construction and $\int_{0}^{t} \phi \mathrm{d} S$ is $\mathcal{F}_{t}$-measurable.

\subsection{Loss Process}

Consider a short position in a claim $X \in \mathcal{C}$ and a trading strategy $\Phi \in \mathcal{S}$. The time- $T$ loss of $X$ that we consider is given by

$$
L_{T}(X, \Phi):=-\left(V_{T}(\phi)-Y\right)
$$

where $V_{T}(\phi)=V_{T}((\phi, 0, \ldots, 0))$ and $Y=X-\sum_{i=1}^{I} u_{i} H_{i}$. If $\overline{\mathbb{Q}}$ calibrates to the market prices of the benchmark instruments, i.e., $\overline{\mathbb{E}}\left[H_{i}\right]=C_{i}^{\star}, i=1, \ldots, I$, then $L_{T}(X, \Phi)=-\left(V_{T}(\Phi)-X\right)$, which corresponds to the overall realized loss from the position. However, if $\overline{\mathbb{Q}}$ does not calibrate perfectly to the benchmark instruments, then there is additional instantaneous P\&L at time 0 from trading the benchmark instruments. This is not included in Eq. (1), and will be ignored in what follows, as this is booked as (sunk) trading cost and as is does not give rise to further risks. 
The goal will be to extend this variable to a loss process $L_{t}(X, \Phi), t<T$, with $\Phi$ a hedging, resp. replicating strategy under $\overline{\mathbb{Q}}$. As both the time- $t$ price, $\overline{\mathbb{E}}\left[Y \mid \mathcal{F}_{t}\right]$ and the strategy $\phi$ are defined only $\overline{\mathbb{Q}}$-a.s., one must be explicit in specifying the version to be used when dealing with models that are not absolutely continuous with respect to $\overline{\mathbb{Q}}$. In our setup, we have $\overline{\mathbb{E}}\left[Y \mid \mathcal{F}_{t}\right]=\overline{\mathbb{E}}\left[Y \mid S_{t}\right]=f\left(S_{t}\right)$ for some Borelmeasurable function $f$, and likewise for the trading strategy. Since $\mathcal{Q}$ expresses the model uncertainty when employing $\overline{\mathbb{Q}}$ for pricing and hedging, it must not be involved in the choice of the respective versions of the pricing and hedging strategies.

Assumption 2 The versions of $\overline{\mathbb{E}}\left[Y \mid S_{t}\right], t \leq T$, and $\phi$ are chosen irrespective of the measures contained in $\mathcal{Q}$.

We further impose linearity conditions on the versions of $\overline{\mathbb{E}}\left[Y \mid \mathcal{F}_{t}\right]$ and $\phi$, which are in general only fulfilled $\overline{\mathbb{Q}}$-a.s. but for all practically relevant models and claims hold for all $\omega \in \Omega$. This will be important for the axiomatic setup in Sect.3.2.

Assumption 3 Let $X_{1}, X_{2} \in \mathcal{C}, \Phi_{1}=\left(\phi_{1}, u_{1}^{1}, \ldots, u_{I}^{1}\right), \Phi_{2}=\left(\phi_{2}, u_{1}^{2}, \ldots, u_{I}^{2}\right) \in$ $\mathcal{S}$ and define $Y_{j}:=X_{j}-\sum_{i=1}^{I} u_{i}^{j} H_{i}, j=1,2$. For all $t \leq T$, it holds that

$$
\overline{\mathbb{E}}\left[a Y_{1}+b Y_{2} \mid \mathcal{F}_{t}\right](\omega)=a \overline{\mathbb{E}}\left[Y_{1} \mid \mathcal{F}_{t}\right](\omega)+b \overline{\mathbb{E}}\left[Y_{2} \mid \mathcal{F}_{t}\right](\omega), \quad a, b \in \mathbb{R}, \quad \omega \in \Omega
$$

and

$$
V_{t}\left(a \phi_{1}(\omega)+b \phi_{2}(\omega)\right)=a V_{t}\left(\phi_{1}(\omega)\right)+b V_{t}\left(\phi_{2}(\omega)\right), \quad a, b \in \mathbb{R}, \quad \omega \in \Omega .
$$

$\overline{\mathbb{E}}\left[Y_{1} \mid S_{T}\right](\omega)=Y_{1}(\omega), \omega \in \Omega$.

Assumptions 2 and 3 will be fulfilled in typical cases relevant in practice. Suppose for example that $S$ is a Black-Scholes model under $\overline{\mathbb{Q}}$. Then prices and the replicating strategy of European payoffs can be determined via the Black-Scholes PDE, and these are suitable versions fulfilling the assumptions.

Definition 1 Let $X \in \mathcal{C}$ and $\Phi=\left(\phi, u_{1}, \ldots, u_{I}\right) \in \mathcal{S}$. The loss process associated with a short position in $X$ and the trading strategy $\Phi$ is given by

$$
\begin{aligned}
L_{t}:=L_{t}(X, \Phi) & =-\left(V_{t}(\phi)-\overline{\mathbb{E}}\left[Y \mid S_{t}\right]\right) \\
& =-\left(V_{0}+\sum_{j=1}^{d} \int_{0}^{t} \phi^{j} \mathrm{~d} S^{j}-\overline{\mathbb{E}}\left[Y \mid S_{t}\right]\right), \quad 0 \leq t \leq T,
\end{aligned}
$$

with $Y=X-\sum_{i=1}^{I} u_{i} H_{i}$ and $V_{0}=\overline{\mathbb{E}}[Y]$.

If $\Phi$ is a replicating strategy under $\overline{\mathbb{Q}}$, then $L_{t}=0 \overline{\mathbb{Q}}$-a.s., but possibly for some $\mathbb{Q} \in \mathcal{Q}, \mathbb{Q}\left(L_{t}=0\right)<1$, which expresses that $\Phi$ fails to replicate $X$ under $\mathbb{Q}$. A model-free hedging strategy is defined as follows:

Definition 2 The trading strategy $\Phi=\left(\left(\phi_{t}\right)_{0 \leq s \leq T}, u_{1}, \ldots, u_{I}\right)$ is a model-free or model-independent replicating strategy for claim $X$ with respect to $\mathcal{Q}$, if $L_{t}=0$, $t \geq 0, \mathbb{Q}-$ a.s., for all $\mathbb{Q} \in \mathcal{Q}$. 
Note that our definition of the hedge error based on a continuous time integral separates model risk from a discretization error. When actually calculating the hedge error, it is necessary to use a time grid small enough such that the discretization error is negligible.

The following proposition shows that the overall expected loss at time $T$ from replicating in $\overline{\mathbb{Q}}$ when the market evolves according to $\mathbb{Q}_{M}$ instead of $\overline{\mathbb{Q}}$ depends only on the price difference.

Proposition 1 1. The total expected loss from replicating under $\overline{\mathbb{Q}}$ claim $X$, that is $\mathbb{E}\left[L_{T}\right]$ plus the initial transaction cost $\mathbb{E}\left[\sum_{i=1}^{I} u_{i}\left(H_{i}^{0}-C_{i}^{\star}\right)\right]$, when the market evolves according to $\mathbb{Q}_{M}$ is just the price difference in the two models, $-(\overline{\mathbb{E}}[X]-$ $\left.\mathbb{E}^{\mathbb{Q}_{M}}[X]\right)$.

2. The price range measure, defined by $\sup _{\mathbb{Q} \in \mathcal{Q}} \mathbb{E}^{\mathbb{Q}}[X]-\inf _{\mathbb{Q} \in \mathcal{Q}} \mathbb{E}^{\mathbb{Q}}[X]$, can be expressed as $\sup _{\mathbb{Q}, \overline{\mathbb{Q}}} \mathbb{E}^{\mathbb{Q}}\left[L_{T}^{\overline{\mathbb{Q}}}\right]$, where $L_{T}^{\overline{\mathbb{Q}}}$ denotes the loss variable from hedging under $\overline{\mathbb{Q}}$.

Proof See [10].

If a claim cannot be replicated, then-given the static hedging component $\sum_{i=1}^{I} u_{i} H_{i}$-a hedging strategy can be defined as a solution $\left(\hat{V}_{0}, \hat{\Phi}\right) \in \mathbb{R} \times \mathcal{S}$ of the optimization problem

$$
\inf _{\left(V_{0} \in \mathbb{R}, \Phi \in \mathcal{S}\right)} \overline{\mathbb{E}}\left[U\left(L_{T}(X, \Phi)\right)\right]=\inf _{\left(V_{0} \in \mathbb{R}, \Phi \in \mathcal{S}\right)} \overline{\mathbb{E}}\left[U\left(-\left(V_{0}+\sum_{j=1}^{d} \int_{0}^{T} \phi \mathrm{d} S^{j}-Y\right)\right)\right],
$$

where $U: \mathbb{R} \rightarrow \mathbb{R}_{+}$weighs the magnitude of the hedge error. The most common choice is $U(x)=x^{2}$, which minimizes the quadratic hedge error. This so-called quadratic hedging has the advantage that the resulting pricing and hedging rules become linear and it is also the analytically most tractable rule. Under this choice of $U(x)$, if $S$ is a martingale, then a solution exists and $\hat{V}_{0}=\overline{\mathbb{E}}[Y],[20]$.

Of course, in an incomplete market, $L_{T}(X, \Phi)$ entails not only losses due to model misspecification, but some losses due to market risk as well, since $\overline{\mathbb{Q}}\left(L_{T}(X, \Phi)=\right.$ $0)<1$, that is, $P \& L$ is incurred even when there is no model uncertainty.

For the explicit determination of $L_{t}(X, \Phi)$ in some examples, we refer to [10]. It is worth noting that in a complete market setup, the loss process corresponds to the tracking error of [15].

\subsection{Loss Distribution}

The next step is to associate a distribution with the loss variable $L_{t}, t \leq T$, based on which risk measures such as value-at-risk and expected shortfall can be defined. 
This is achieved by considering an extended probability space $(\Omega, \mathcal{F}, \mathbb{P})$, where $\mathcal{F}$ now incorporates in addition the model uncertainty and $\mathbb{P}$ contains information about the degree of uncertainty associated with each model. To make this precise, let $\mathcal{G} \subset \mathcal{F}$ be a $\sigma$-algebra such that conditioning on $\mathcal{G}$ eliminates the uncertainty about the pricing measure $\mathbb{Q} \in \mathcal{Q}$. In this setting, the measures in $\mathcal{Q}$ constitute a regular conditional probability with respect to $\mathcal{G}$. For existence and construction of this probability space, we refer to [10].

In this setup, the models can be indexed by a random variable $\theta \in \Theta \subseteq \mathbb{R}$, with $\sigma(\theta)=\mathcal{G}$, so that $\mathbb{Q}_{\theta}=\mathbb{P}(\cdot \mid \sigma(\theta))$ and

$$
\mathbb{P}(B)=\mathbb{E}[\mathbb{P}(B \mid \sigma(\theta))]=\int_{\Omega} \mathbb{P}(B \mid \sigma(\theta)) \mathrm{d} \mathbb{P}=\int_{\Theta} \mathbb{P}(B \mid \theta=a) \mu(\mathrm{d} a), \quad B \in \mathcal{F}
$$

where $\mu$ is the distribution of $\theta$. In particular, losses from hedging in a misspecified model under model uncertainty have distribution function

$$
\mathbb{P}\left(L_{t} \leq x\right)=\int_{\Theta} \mathbb{Q}_{a}\left(L_{t} \leq x\right) \mu(\mathrm{d} a), \quad 0 \leq t \leq T .
$$

The following proposition is proved in [10].

Proposition 2 A strategy $\Phi$ is a model-free hedging strategy for claim X $\mathbb{P}$-a.s. if and only if $\mathbb{P}\left(L_{t}=0\right)=1$.

Hence, model uncertainty is expressed by the unconditional distribution $\mathbb{P}$, whereas model certainty is expressed via the conditional distribution $\mathbb{P}(\cdot \mid \sigma(\theta))$.

A concrete approach to determining the distribution $\theta$ is presented in [10]. Here, probability weights are assigned to the models in $\mathcal{Q}$ via the Akaike Information Criterion (AIC), e.g., [6, 7], which trades off calibration quality against model complexity.

\section{Measures of Model Risk}

The loss distribution aggregated across the measures in $\mathcal{Q}$ from Sect. 2.3 is the key input to define measures of model risk. For the time being, we continue to work in a setting where a particular model $\overline{\mathbb{Q}}$ is used for pricing and hedging, as this is appropriately quantifies the model risk from a bank's internal perspective.

If a claim cannot be replicated, and the trading strategy $\Phi$ is merely a hedging strategy in some risk-minimizing sense, then the loss variable $L_{t}(X, \Phi)$ from Definition 1 features not only model risk, but also the unhedged market risk. To disentangle model risk from the market risk, one could first determine the market risk from the unhedged part of the claim under $\overline{\mathbb{Q}}$ and set this into relation to the overall residual risk. This requires taking into account potential diversification effects, since 
risks are not additive. We shall continue to work under the setup of measuring residual risk, and use the terminology "model risk," although some market risk is also present.

Market incompleteness can also be seen to be a form of model risk, as-in addition to the uncertainty on the objective measure-it causes uncertainty on the equivalent martingale measure. However, hedging strategies would typically be chosen that are risk minimizing not under the martingale measure, but risk minimizing under the objective measure. In the case of continuous asset prices, this implies that hedging is done under the minimal-martingale measure, which is uniquely determined. In practice, it is more common to choose an equivalent measure that calibrates sufficiently well, and in this case one could argue that incompleteness also increases model uncertainty. In our setup, this would be reflected by a larger set $\mathcal{Q}$.

\subsection{Value-at-Risk and Expected Shortfall}

The usual value-at-risk and expected shortfall measures are defined as follows:

Definition 3 Let $L_{t}(X, \Phi)$ be the time- $t$ loss from the strategy $\Phi$ that hedges claim $X$ under $\overline{\mathbb{Q}}$. Given a confidence level $\alpha \in(0,1)$,

1. Value-at-risk $(\operatorname{VaR})$ is given by

$$
\operatorname{VaR}_{\alpha}\left(L_{t}(X, \Phi)\right)=\inf \left\{l \in \mathbb{R}: \mathbb{P}\left(L_{t}(X, \Phi)>l\right) \leq 1-\alpha\right\}
$$

that is, $\mathrm{VaR}_{\alpha}$ is just the $\alpha$-quantile of the loss distribution;

2. Expected shortfall (ES) is given by

$$
\operatorname{ES}_{\alpha}\left(L_{t}(X, \Phi)\right)=\frac{1}{(1-\alpha)} \int_{\alpha}^{1} \operatorname{VaR}_{u}\left(L_{t}(X, \Phi)\right) \mathrm{d} u
$$

In the presence of benchmark instruments, the hedging strategy in model $\overline{\mathbb{Q}}$ may not be unique. If the claim $X$ can be replicated, then $\Pi=\left\{\Phi \in \mathcal{S}: \overline{\mathbb{Q}}\left(L_{t}(X, \Phi)=\right.\right.$ $0)=1, t \leq T\}$ is the set of replicating strategies for claim $X$ in model $\overline{\mathbb{Q}}$. Otherwise, we focus on quadratic hedging and define $\Pi=\left\{\Phi=\left(\phi^{0}, \ldots, \phi^{j}, u_{1}, \ldots, u_{I}\right) \in\right.$ $\mathcal{S},\left(u_{1}, \ldots, u_{I}\right) \in \mathbb{R}^{I}: \Phi=\hat{\Phi}$ under $\left.\overline{\mathbb{Q}}\right\}$, where $\hat{\Phi}$ refers to the quadratic riskminimizing strategy attaining the infimum in (3) with $U(x)=x^{2}$. Because in an incomplete market, the loss from hedging entails some market risk aside from model risk, the benchmark instruments play a more important role than in complete market, as they are not necessarily redundant, but may reduce the hedge error under $\overline{\mathbb{Q}}$.

To abstract from the particular hedging strategy chosen, we define measures that quantify the minimal degree of model dependence, indicating that when pricing and hedging under measure $\overline{\mathbb{Q}}$, the model dependence cannot be further reduced. This is 
reasonable in the sense that it is not of interest whether a position is indeed hedged or not. Rather the hedging argument serves only to eliminate (or reduce, in case the claim cannot be replicated) P\&L from market risk. Choosing the minimal degree allows to appropriately capture claims that can be replicated in a model-free way.

Definition 4 Concrete measures capturing the model uncertainty when pricing and hedging claim $X$ according to model $\overline{\mathbb{Q}}$ are given by

1. $\mu_{\mathrm{SQE}, t}^{\overline{\mathbb{Q}}}(X)=\inf _{\Phi \in \Pi} \mathbb{E}\left[L_{t}(X, \Phi)^{2}\right]$,

2. $\mu_{\mathrm{VaR}, \alpha, t}^{\overline{\mathbb{Q}}}(X)=\inf _{\Phi \in \Pi} \operatorname{VaR}_{\alpha}\left(\left|L_{t}(X, \Phi)\right|\right)$,

3. $\mu_{\mathrm{ES}, \alpha, t}^{\overline{\mathbb{Q}}}(X)=\inf _{\Phi \in \Pi} \operatorname{ES}_{\alpha}\left(\left|L_{t}(X, \Phi)\right|\right)$.

4. $\rho_{\mathrm{VaR}, \alpha, t}^{\overline{\mathbb{Q}}}(X)=\inf _{\Phi \in \Pi} \max \left(\operatorname{VaR}_{\alpha}\left(L_{t}(X, \Phi)\right), 0\right)$,

5. $\rho_{\mathrm{ES}, \alpha, t}^{\overline{\mathbb{Q}}}(X)=\inf _{\Phi \in \Pi} \max \left(\operatorname{ES}_{\alpha}\left(L_{t}(X, \Phi)\right), 0\right)$.

The measures $\mu_{\mathrm{VaR}, \alpha, t}^{\overline{\mathbb{Q}}}$ and $\mu_{\mathrm{ES}, \alpha, t}^{\overline{\mathbb{Q}}}$ capture model uncertainty in an absolute sense, and are thus measures of the magnitude or degree of model uncertainty. The measures $\rho_{\mathrm{VaR}, \alpha, t}^{\overline{\mathbb{Q}}}$ and $\rho_{\mathrm{ES}, \alpha, t}^{\overline{\mathbb{Q}}}$ consider losses only. As such, they are suitable for defining a capital charge against losses from model risk.

Contrary to the case of bank internal risk measurement, a regulator may wish to measure model risk independently of a particular pricing or hedging measure, taking a more prudent approach. To abstract from the pricing measure, one would first define the set $\mathcal{Q}_{H} \subseteq \mathcal{Q}$ of potential pricing and hedging measures (e.g., measures that calibrate sufficiently well) and then define the risk measure in a worst-case sense as follows:

Definition 5 Let $\mu_{t}^{\mathbb{Q}_{H}}(X)$ be a measure of model uncertainty when pricing and hedging $X$ according to model $\mathbb{Q}_{H} \in \mathcal{Q}_{H}$. The model uncertainty of claim $X$ is given by

$$
\mu_{t}(X)=\sup _{\mathbb{Q}_{H} \in \mathcal{Q}_{H}} \mu_{t}^{\mathbb{Q}_{H}}(X)
$$

Capital charges can then be determined from either $\mu_{\mathrm{VaR}, \alpha, t}^{\overline{\mathbb{Q}}}(X)$, resp. $\mu_{\mathrm{ES}, \alpha, t}^{\overline{\mathbb{Q}}}(X)$, or from $\mu_{\mathrm{VaR}, \alpha, t}(X)$, resp. $\mu_{\mathrm{ES}, \alpha, t}(X)$.

\subsection{Axioms for Measures of Model Risk}

Cont [8] introduces a set of axioms for measures of model risk. A measure satisfying these axioms is called a convex measure of model risk. The axioms follow the general notion of convex risk measures, $[18,21]$, but are adapted to the special case of model risk. In particular, these axioms take into account the possibility of static hedging 
with liquidly traded option and of hedging in a model-free way. More specifically, the axioms postulate that an option that can be statically hedged with liquidly traded options is assigned a model risk bounded by the cost of replication, which can be expressed in terms of the bid-ask spread. Consequently, partial static hedging for a claim reduces model risk. Further, the possibility of model-free hedging with the underlying asset reduces model risk to zero. Finally, to express that model risk can be reduced through diversification, convexity is required.

Here we only state the following result, which ensures that our measures fulfill the axioms proposed in Cont [8]. The proof is given in [10] for complete markets and can be easily generalized to an incomplete market.

Proposition 3 The measures $\mu_{\mathrm{SQE}, t}^{\overline{\mathbb{Q}}}(X), \mu_{\mathrm{ES}, \alpha, t}^{\overline{\mathbb{Q}}}(X)$ and $\rho_{\mathrm{ES}, \alpha, t}^{\overline{\mathbb{Q}}}(X)$ satisfy the axioms of model uncertainty. The measures $\mu_{\mathrm{VaR}, \alpha, t}^{\overline{\mathbb{Q}}}(X)$ and $\rho_{\mathrm{VaR}, \alpha, t}^{\overline{\mathbb{Q}}}(X)$ satisfy Axioms 1, 2, and 4.

\section{Hedge Differences}

Instead of considering the P\&L arising from model misspecification as in Sect. 2.2, one might be interested in a direct comparison of hedging strategies implied by different models. For example, one might wish to assess the quality of hedging strategies determined from a deliberately misspecified, but simpler model, in a more appropriate, but more involved model.

We first explain the idea with respect to one alternative model $\mathbb{Q}_{M} \in \mathcal{Q}$ and outline then how measures with respect to the entire model set can be built. As before, $\overline{\mathbb{Q}}$ is the model for pricing and hedging and, fixing a claim $X \in \mathcal{C}, \Pi$ is the set of quadratic risk-minimizing $(\mathrm{QRM})$ hedging strategies for $X$ under $\overline{\mathbb{Q}}$ (containing various hedging strategies, depending on how static hedges with the benchmark instruments are chosen).

We seek an answer to the following question: If the market turns out to follow $\mathbb{Q}_{M}$, what is the loss incurred by hedging in $\overline{\mathbb{Q}}$ instead of hedging in $\mathbb{Q}_{M}$ ? Let $\Phi=\left(\phi, u_{1}, \ldots, u_{I}\right) \in \Pi$ be the QRM strategy for $Y=X-\sum_{i=1}^{I} u_{i} H_{i}$, and let $\Phi_{M}$ be the respective QRM strategy for $Y$ derived under $\mathbb{Q}_{M}$. The relative difference of the hedge portfolio compared to the hedge portfolio when using the strategy of $\mathbb{Q}_{M}$ is given by

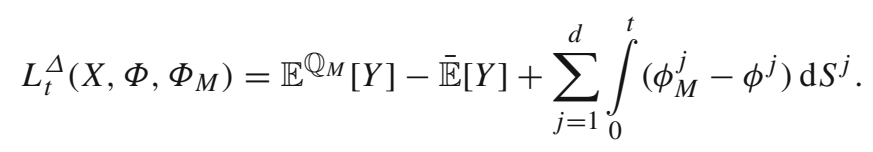


This variable differs from $L_{t}(X, \Phi)$, cf. Eq. (2), in that it expresses the difference between the hedging strategies $\Phi$ and $\Phi_{M}$, whereas $L_{t}(X, \Phi)$ describes the difference between the hedging strategy $\Phi$ and the claim $X{ }^{1}$

The next proposition provides some insight on the different nature of the two variables.

Proposition 4 The following properties hold for the processes $L^{\Delta}\left(X, \Phi, \Phi_{M}\right)$ and $L(X, \Phi)$ :

1. $L^{\Delta}\left(X, \Phi, \Phi_{M}\right)$ is a $\mathbb{Q}_{M}$-martingale with $L_{0}^{\Delta}\left(X, \Phi, \Phi_{M}\right)=\mathbb{E}^{\mathbb{Q}_{M}[Y]-\overline{\mathbb{E}}[Y]}$

2. $\mathbb{E}^{\mathbb{Q}_{M}}\left[L_{T}(X, \Phi)\right]=\mathbb{E}^{\mathbb{Q}_{M}[Y]-\bar{E}[Y]}$

3. $L_{T}^{\Delta}\left(X, \Phi, \Phi_{M}\right)=L_{T}(X, \Phi) \mathbb{Q}_{M}$-a.s. if $Y$ can be replicated under $\mathbb{Q}_{M}$

4. $L_{t}^{\Delta}\left(X, \Phi, \Phi_{M}\right)-L_{t}(X, \Phi)=\mathbb{E}^{\mathbb{Q}_{M}}\left[Y \mid \mathcal{F}_{t}\right]-\overline{\mathbb{E}}\left[Y \mid \mathcal{F}_{t}\right] \mathbb{Q}_{M}-$ a.s. if $Y$ can be replicated under $\mathbb{Q}_{M}$.

Proof 1. This follows directly from the definition of $L^{\Delta}\left(X, \Phi, \Phi_{M}\right)$ and the fact that $\Phi^{M}$ and $\Phi$ are in $\mathcal{S}$.

2. See Proposition 1.

3. If $Y$ can be replicated, then $Y=\mathbb{E}^{\mathbb{Q}_{M}}[Y]+\sum_{j=1}^{d} \int_{0}^{T} \phi_{M}^{j} \mathrm{~d} S^{j} \mathbb{Q}_{M}$-a.s., and consequently $L_{T}^{\Delta}\left(X, \Phi, \Phi_{M}\right)=Y-\left(\overline{\mathbb{E}}[Y]+\sum_{j=1}^{d} \int_{0}^{T} \phi^{j} \mathrm{~d} S^{j}\right) \mathbb{Q}_{M}$-a.s.. The claim follows by observing that $L_{t}(X, \Phi)=-\left(\overline{\mathbb{E}}[Y]+\sum_{j=1}^{d} \int_{0}^{T} \phi^{j} \mathrm{~d} S^{j}-Y\right)$.

4. Using that $L_{t}^{\Delta}\left(X, \Phi, \Phi_{M}\right)=\mathbb{E}^{\mathbb{Q}_{M}}\left[Y \mid \mathcal{F}_{t}\right]-\left(\overline{\mathbb{E}}[Y]+\sum_{j=1}^{d} \int_{0}^{t} \phi^{j} \mathrm{~d} S^{j} \mathbb{Q}_{M}\right.$-a.s., since $Y$ can be replicated under $\mathbb{Q}_{M}$, the claim follows with the definition of $L_{t}(X, \Phi)$.

Observe that the variable $L_{t}(X, \Phi)$ is neither a sub-martingale nor a super-martingale as shown in the example in [10, Sect. 3.5.].

As an example, Fig. 1 shows the distributions of $L_{t}(X, \Phi)$ and $L_{t}^{\Delta}\left(X, \Phi, \Phi_{M}\right)$ for an at-the-money call option $X=\left(S_{T}-K\right)^{+}$with $S_{0}=K=1$, with expiry $T$ in 3 months, at time $t=T / 2$, dynamically hedged with the underlying asset, i.e., $\Phi=(\phi)$, resp. $\Phi_{M}=\left(\phi_{M}\right)$. Under the misspecified model $\overline{\mathbb{Q}}$, the asset price process corresponds to a geometric Brownian motion with $20 \%$ volatility, whereas under $\mathbb{Q}_{M}$ the asset price process follows a geometric Brownian motion with $25 \%$ volatility. The correlation of the two loss variables is $67.97 \%$. At maturity $T$, both variables agree.

Generalizing the relative hedge difference to a set of models is not straightforward, as the loss variable $L_{t}^{\Delta}\left(X, \Phi, \Phi_{M}\right)$ depends explicitly on $\mathbb{Q}_{M}$ and, as such, a version of the variable that is valid under all models cannot be constructed. [12] shows how a loss distribution under model uncertainty can be constructed, which can then be used to define the usual risk measures such as value-at-risk and expected shortfall.

\footnotetext{
1 There is no need to pose specific conditions on the version of the hedging strategy $\Phi_{M}$ chosen, since in the following only properties of $L_{t}^{\Delta}\left(X, \Phi, \Phi_{M}\right)$ under $\mathbb{Q}_{M}$ are analyzed.
} 

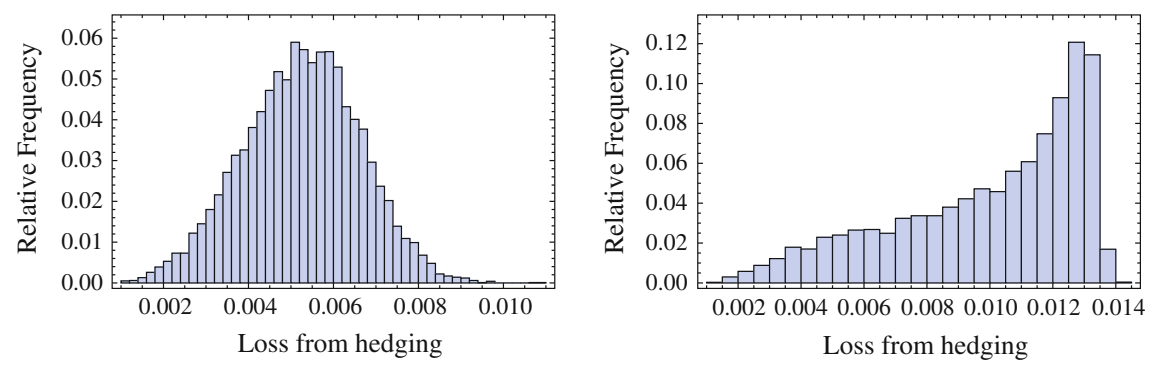

Fig. 1 Loss at $t=1 / 2 T$ from dynamically hedging an at-the-money call option with a maturity $T$ of 3 months based on 10,000 simulations and 1,000 time steps. Left Distribution of $L_{t}(X, \Phi)$, $\mathbb{E}\left[L_{t}(X, \Phi)\right]=0.0053$. Right Distribution of $L_{t}^{\Delta}\left(X, \Phi, \Phi_{M}\right), \mathbb{E}\left[L_{t}^{\Delta}\left(X, \Phi, \Phi_{M}\right)\right]=0.0099$, which equals the initial price difference

\section{Application to Energy Markets}

As a real-worked example, we study the loss variables and risks from hedging options on futures in energy markets. The spot and future prices in energy markets are extremely volatile and show large spikes, and a realistic model for the price dynamics should therefore involve jumps. However, continuous models based on Brownian motions are not only computationally more tractable, but prevalent in practice. Our analysis sheds light on the risks of hedging in a simplified continuous model instead of a model involving jumps.

Assume given a probability space $\left(\Omega,\left(\mathcal{F}_{t}\right)_{0 \leq t \leq T}\right)$ with a measure $\tilde{\mathbb{P}}$ on which a two-dimensional Lévy process $\left(L_{t}\right)=\left(L_{1, t}, L_{2, t}\right)_{t>0}$ with independent components is defined. A popular two-factor model for the energy spot price is developed by Schwartz and Smith [27]. The spot is driven by a short-term mean reverting factor to account for short-term energy supply and energy demand and a long-term factor for changes in the equilibrium price level. In its extended form, [4, Sect. 5], the logarithm of the spot price is

$$
\log S_{t}=\Lambda_{t}+X_{t}+Y_{t}
$$

with $\left(\Lambda_{t}\right)_{t>0}$ a deterministic seasonality function, $\left(X_{t}\right)_{t>0}$ a Lévy driven Ornstein Uhlenbeck process with dynamics $\mathrm{d} X_{t}=-\lambda X_{t} \mathrm{~d} t+\mathrm{d} L_{1, t}$ and $\left(Y_{t}\right)_{t>0}$ defined by $\mathrm{d} Y_{t}=\mathrm{d} L_{2, t}$. We further assume that the cumulant function $\Psi(z):=\log \left(\mathbb{E}\left[e^{\left\langle z, L_{1}\right\rangle}\right]\right)$ is well defined for $z=\left(z_{1}, z_{2}\right) \in \mathbb{R}^{2},|z| \leq C$, for $C \in \mathbb{R}$. Due to the independence of $L_{1}$ and $L_{2}$, the cumulant transforms of both processes add up and we have $\Psi(z)=$ $\Psi_{1}\left(z_{1}\right)+\Psi_{2}\left(z_{2}\right)$ where $\Psi_{1}$ and $\Psi_{2}$ is the cumulant for $L_{1}$ and $L_{2}$, respectively.

We consider the pricing and hedging of options on the future contract. In contrast to, for example, equity markets, the future contract in energy delivers over a period of time $\left[T_{1}, T_{2}\right]$ instead of a fixed time point by defining a payout 


$$
\frac{1}{T_{2}-T_{1}} \int_{T_{1}}^{T_{2}} S_{r} \mathrm{~d} r
$$

in return for the agreed future price. While the spot is not tradable due to lack of storage opportunities, the future is tradable and used for hedging both options on the future itself and options directly on the spot price. Assuming that the future price $F_{t}$ equals its expected payout

$$
F_{t}=\mathbb{E}^{\mathbb{Q}}\left[\frac{1}{T_{2}-T_{1}} \int_{T_{1}}^{T_{2}} S_{r} \mathrm{~d} r \mid \mathcal{F}_{t}\right]
$$

under a pricing measure $\mathbb{Q} \sim \tilde{\mathbb{P}}$, the value $F_{t}$ is derived in analytic form in [4]. Under the assumption that $L_{1}$ and $L_{2}$ are normal inverse Gaussian (NIG) distributed Lévy processes an approximate process $\left(\widehat{F}_{t}^{L}\right)_{t<T_{2}}$ is determined in [4] by matching first and second moments such that $\left(\widehat{F}_{t}^{L}\right)_{t<T_{2}}$ is of exponential additive type. We assume in this application that $\mathbb{Q}=\tilde{\mathbb{P}}$. The value of $\widehat{F}_{t}^{L}$ is then

$$
\widehat{F}_{t}^{L}=F_{0} \exp \left(-\int_{0}^{t} \Psi_{1}\left(\Sigma_{1}^{L}(s)\right)+\Psi_{2}\left(\Sigma_{2}^{L}(s)\right) \mathrm{d} s+\int_{0}^{t} \Sigma_{1}^{L}(s) \mathrm{d} L_{1, s}+\int_{0}^{t} \Sigma_{2}^{L}(s) \mathrm{d} L_{2, s}\right)
$$

with time-dependent, deterministic functions $\Sigma_{1}^{L}(t)$ and $\Sigma_{2}^{L}(t)$. The process $\widehat{F}^{L}$ depends on the interval $\left[T_{1}, T_{2}\right]$, but in order to avoid overloading the notation and since we shall only consider a single delivery period in our example, we simply write $\widehat{F}^{L}, \Sigma_{1}^{L}$ and $\Sigma_{2}^{L}$. The market under this model is incomplete and claims can in general only be hedged with risk-minimizing strategies. Integral representations for prices and quadratic risk-minimizing hedge positions of call and put payoffs can be derived, and we refer the reader to [4, Prop.3.9.] for further details and the explicit formulas.

As a pricing and hedging model, we consider a simplified version of (6), which is driven by two (nonstandard) independent $\overline{\mathbb{Q}}$-Brownian motions $\left(B_{1, t}\right)_{t>0}$ and $\left(B_{2, t}\right)_{t>0}$ defined on $\left(\Omega,\left(\mathcal{F}_{t}\right)_{0 \leq t \leq T}\right)$ and we derive, again by moment matching, an analog approximate future price process $\widehat{F}^{B}$ of the form

$$
\widehat{F}_{t}^{B}=F_{0} \exp \left(-\int_{0}^{t} \Psi_{1}^{B}\left(\left(\Sigma_{1}^{B}(s)\right)+\Psi_{2}^{B}\left(\left(\Sigma_{2}^{B}(s)\right) \mathrm{d} s+\int_{0}^{t} \Sigma_{1}^{B}(s) \mathrm{d} B_{1, s}+\int_{0}^{t} \Sigma_{2}^{B}(s) \mathrm{d} B_{2, s}\right)\right.\right.
$$

with time-dependent, deterministic functions $\Sigma_{1}^{B}(t)$ and $\Sigma_{2}^{B}(t)$ and with $\Psi_{1}^{B}(z)$ and $\Psi_{2}^{B}(z)$ being the cumulant transforms of $B_{1,1}$ and $B_{2,1}$. 
Although the model has two sources of randomness, it is a complete model under the filtration generated by the future price itself as the next proposition shows, which means that all practically relevant claims can be replicated.

Proposition 5 Let $\left(\mathcal{G}_{t}\right)_{t<T}$ be the filtration generated by $\widehat{F}^{B}$ up to time t, i.e., $\mathcal{G}_{t}:=$ $\sigma\left\{\widehat{F}_{s}^{B}, s \leq t\right\}$. Then the market consisting of $\widehat{F}^{B}$ and a constant riskless bank account is a complete financial market with respect to $\left(\mathcal{G}_{t}\right)_{t<T}$.

\section{Proof See [12].}

We estimate the parameters for both models based on future and spot data from Nord Pool energy exchange. We use average daily system peak load electricity spot prices for the period from January 2011 until May 2013 (prices as shown on Bloomberg page "ENOSOSPK") and weekday prices for front month and second month future contracts. For details on the estimation procedure, we refer to [4, Sect. 5.2.]. In Table 1, we collect the parameter estimates for the two factors of both models, the simplified model with two nonstandard Brownian motions and the model with two independent NIG-Lévy processes. The estimates for the Brownian factor are only the drift term $\mu$ and the volatility term $\sigma$. The NIG distribution is a four-parameter distribution with scale parameter $\delta$, tail heaviness $\alpha$, skew parameter $\beta$, and the location parameter $v$, see [3].

Figure 2 shows the empirical return distributions of both factors together with the density function of the estimated distribution. It is obvious that the NIG distribution provides a significantly better fit to the empirical returns than the normal distribution.

The claim to be hedged is an option on a future with a one-week delivery period trading one month prior to expiry, so that $T_{1}=23$ and $T_{2}=30$. Based on the parameter estimates, we determine scaling terms $\Sigma_{1}^{L}(t)$ and $\Sigma_{2}^{L}(t)$ for the dynamics of $\widehat{F}_{t}^{L}$ and scaling terms $\Sigma_{1}^{B}(t)$ and $\Sigma_{2}^{B}(t)$ for the dynamics of $\widehat{F}^{B}$, respectively. Assuming that the measures $\mathbb{Q}$ and $\overline{\mathbb{Q}}$ are orthogonal, we define an aggregating process $\widehat{F}$ such that $\widehat{F}=\widehat{F}^{B} \widehat{\mathbb{Q}}$-a.s. and $\widehat{F}=\widehat{F}_{t}^{L} \mathbb{Q}$-a.s.. Pricing and hedging is performed under $\overline{\mathbb{Q}}$, and there is only one alternative measure, denoted by $\mathbb{Q}$. Our model set is thus $\mathcal{Q}=\{\overline{\mathbb{Q}}, \mathbb{Q}\}$. Applying the Akaike Information Criterion (AIC), we assign a probability distribution to the model set $\mathcal{Q}$. It turns out that model $\mathbb{Q}$ gets assigned a probability of basically 1 due to its much better fit of the returns and we simulate according to this model.

We consider an at-the-money call option $X:=\left(\widehat{F}_{T_{2}}-F_{0}\right)^{+}$and calculate the hedge positions implied by $\overline{\mathbb{Q}}$. For the simulation of the process under $\mathbb{Q}$, we use 600 time steps in order to reduce the discretization error. We investigate the distribution of $L_{T}^{\Delta}\left(X, \Phi, \Phi_{\mathbb{Q}}\right)$ and $L_{T}(X, \Phi)$, with $\Phi$ and $\Phi_{\mathbb{Q}}$ dynamic hedging strategy as there are no benchmark instruments. As implied by Proposition 5, the hedging strategy is actually a perfect hedge under the model $\overline{\mathbb{Q}}$.

Figure 3 shows on the left-hand side the distributions under $\mathbb{Q}$ of $L_{T}(X, \Phi)$ and $L_{T}^{\Delta}\left(X, \Phi, \Phi_{\mathbb{Q}}\right)$. To compare, Fig. 3 shows the distribution under $\mathbb{Q}$ of the hedge error $L_{T}\left(X, \Phi^{\mathbb{Q}}\right)$ when hedging under $\mathbb{Q}$ (top right). Here, the hedge error is introduced by market incompleteness. 
Table 1 Estimated parameters for the NIG distributions of $L_{1, t}$ and $L_{2, t}$ and parameters for the normal distributions of $B_{1, t}$ and $B_{2, t}$

\begin{tabular}{l|l|l|r|l}
\hline & $\hat{\alpha}$ & $\hat{\beta}$ & $\hat{v}$ & $\hat{\delta}$ \\
\hline$L_{2, t}$ & 1.9240 & -0.8860 & 0.0176 & 0.0622 \\
$L_{1, t}$ & 33.3008 & -1.0988 & -0.0009 & 0.0071 \\
\hline & $\hat{\sigma}$ & $\hat{\mu}$ & & \\
\hline$B_{2, t}$ & 0.2328 & -0.0004 & & \\
$B_{1, t}$ & 0.0133 & 0.0002 & & \\
\hline
\end{tabular}
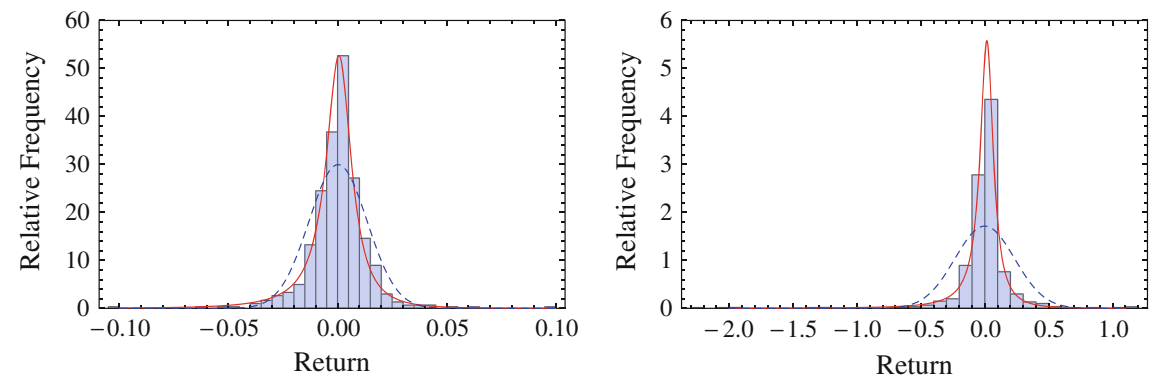

Fig. 2 Empirical distributions of long-term factor (left) and short-term factor (right) together with fitted NIG distribution (solid line) and normal distribution (dashed line)

It turns out that the loss due to the misspecified model $\overline{\mathbb{Q}}$ is minor compared to the loss due to the incompleteness. The loss due to model misspecification as measured by $L_{T}^{\Delta}\left(X, \Phi, \Phi_{\mathbb{Q}}\right)$ has a mean-squared value of $\mu_{\mathrm{SQE}, t}^{\overline{\mathbb{Q}}, \Delta}(X)=\mathbb{E}^{\mathbb{Q}}\left[\left(L_{T}^{\Delta}\left(X, \Phi, \Phi_{\mathbb{Q}}\right)\right)^{2}\right]=$ 9.50. The mean-squared hedge error from hedging under the misspecified model is greater with $\mu_{\mathrm{SQE}, t}^{\overline{\mathbb{Q}}}(X)=\mathbb{E}^{\mathbb{Q}}\left[\left(L_{T}(X, \Phi)\right)^{2}\right]=34.61$. Although the magnitude appears high, it is relativized by the fact that even under correct model specification the mean-squared hedge error $\mathbb{E}^{\mathbb{Q}}\left[\left(L_{T}\left(X, \Phi_{\mathbb{Q}}\right)\right)^{2}\right]$ is 25.54 . The initial prices under the two models are $\mathbb{E}^{\overline{\mathbb{Q}}}[X]=10.954$ and $\mathbb{E}^{\mathbb{Q}}[X]=8.068$, respectively. If we consider the variance of the loss variables, which corrects for the mean, it turns out that the impact from the misspecified hedge is rather low. For the variable $L_{T}^{\Delta}\left(X, \Phi, \Phi_{\mathbb{Q}}\right)$, we get $\operatorname{Var}\left(L_{T}^{\Delta}\left(X, \Phi, \Phi_{\mathbb{Q}}\right)\right)=1.07$. We find that $\operatorname{Var}\left(L_{T}(X, \Phi)\right)$ and $\mathbb{E}^{\mathbb{Q}}\left[\left(L_{T}\left(X, \Phi_{\mathbb{Q}}\right)\right)^{2}\right]=\operatorname{Var}\left(L_{T}\left(X, \Phi_{\mathbb{Q}}\right)\right)$ are similar with 25.71 and 25.56, respectively. The lower right of Fig. 3 shows a scatter plot of $\left(L_{T}(X, \Phi)\right.$ and $L_{T}\left(X, \Phi_{\mathbb{Q}}\right)$. The two variables show a correlation of $97.91 \%$, implying a strong linear dependence between the hedge error under model $\mathbb{Q}$ (market risk) and the hedge error due to using the misspecified model $\overline{\mathbb{Q}}$.

The fact that the impact due to hedging in the wrong model is relatively low in this case study should not be misinterpreted. It confirms a stylized fact that is well known for diffusion processes (see [15]), namely that, hedging is robust, as long as the overall variance of the underlying is described sufficiently well by the model. 

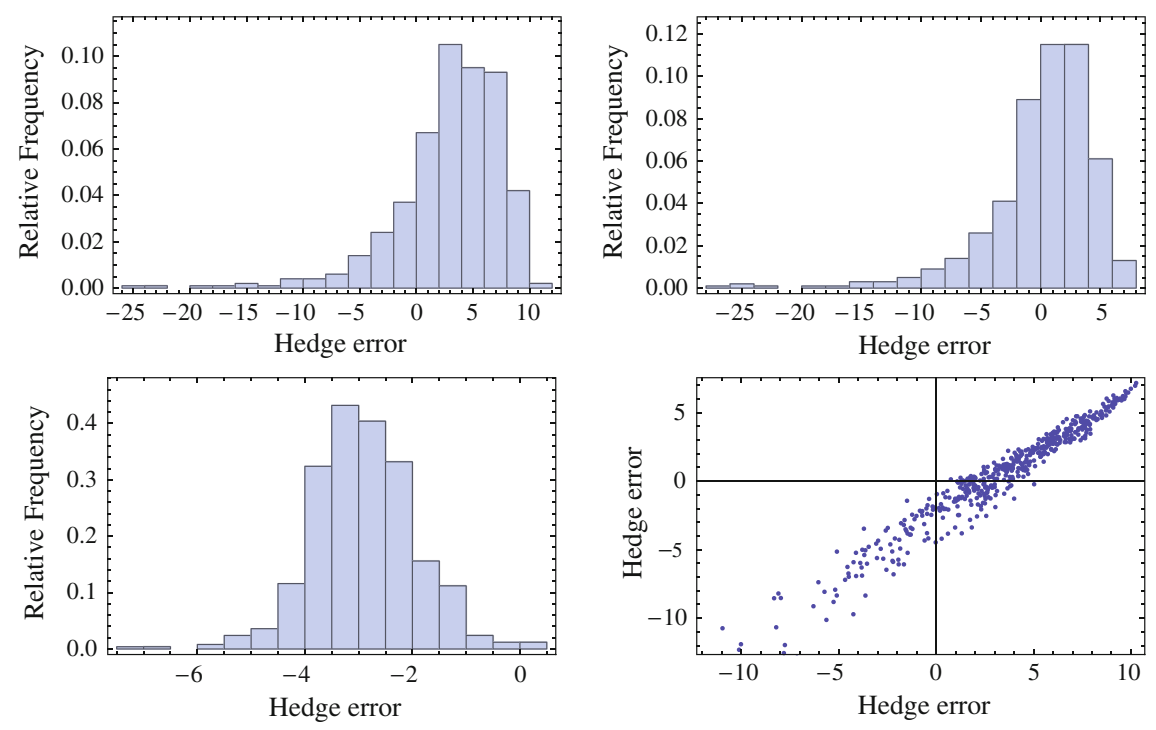

Fig. 3 Upper left $\mathbb{Q}\left(L_{T}(X, \Phi)<\cdot\right)$. Upper right $\mathbb{Q}\left(L_{T}\left(X, \Phi_{\mathbb{Q}}\right)<\cdot\right)$. Lower left $\mathbb{Q}\left(L_{T}^{\Delta}(X, \Phi\right.$, $\left.\left.\Phi_{\mathbb{Q}}\right)<\cdot\right)$. Lower right Scatter plot of $L_{T}(X, \Phi)$ and $L_{T}\left(X, \Phi_{\mathbb{Q}}\right)$

The overall volatility in our setup is the same for both models due to the moment matching procedure and uncertainty in this volatility is likely to result in greater model risk. The study makes also clear that the hedging error due to incompleteness cannot be neglected.

Open Access This chapter is distributed under the terms of the Creative Commons Attribution Noncommercial License, which permits any noncommercial use, distribution, and reproduction in any medium, provided the original author(s) and source are credited.

\section{References}

1. Artzner, P., Delbaen, F., Eber, J., Heath, D.: Coherent measures of risk. Math. Financ. 9(3), 203-228 (1999)

2. Bannör, K., Scherer, M.: Capturing parameter uncertainty with convex risk measures. Eur. Actuar. J. 3, 97-132 (2013)

3. Barndorff-Nielsen, O.E.: Processes of normal inverse Gaussian type. Financ. Stochast. 2, 41-68 (1998)

4. Benth, F.E., Detering, N.: Pricing and hedging Asian-style options in energy. Financ. Stochast. (2013)

5. BIS: Revisions to the Basel II market risk framework. Basel committee on banking supervision, Bank for International Settlements, February (2011)

6. Burnham, K., Anderson, D.: Model Selection and Multimodel Inference: A Practical Information-Theoretic Approach, 2nd edn. Springer, New York (2002) 
7. Burnham, K., Anderson, D.: Multimodel inference-understanding AIC and BIC in model selection. Sociol. Methods Res. 33(2), 261-304 (2004)

8. Cont, R.: Model uncertainty and its impact on the pricing of derivative instruments. Math. Financ. 16(3), 519-547 (2006)

9. Denis, L., Martini, C.: A theoretical framework for the pricing of contingent claims in the presence of model uncertainty. Ann. Appl. Probab. 16(2), 827-852 (2006)

10. Detering, N., Packham, N.: Measuring the model risk of contingent claims. Working Paper, Frankfurt School of Finance \& Management (submitted) (2013)

11. Detering, N., Weber, A., Wystup, U.: Return distributions of equity-linked retirement plans under jump and interest rate risk. Eur. Actuar. J. 3(1), 203-228 (2013)

12. Detering, N.: Measuring the model risk of quadratic risk minimizing hedging strategies with an application to energy markets. Working paper, February (2014)

13. EBA: Discussion paper on draft regulatory technical standards on prudent valuation, under Article 100 of the draft Capital Requirements Regulation (CRR). Discussion Paper, European Banking Authority, November (2012)

14. El Karoui, N., Quenez, M.: Dynamic programming and pricing of contingent claims in an incomplete market. SIAM J. Control Optim. 33(1), 29-66 (1995)

15. El Karoui, N., Jeanblanc-Picqué, M., Shreve, S.: Robustness of the Black and Scholes formula. Math. Financ. 8(2), 93-126 (1998)

16. Epstein, L.: A definition of uncertainty aversion. Rev. Econ. Stud. 66(3), 579-608 (1999)

17. Federal Reserve: Supervisory guidance on model risk management. Board of Governors of the Federal Reserve System, Office of the Comptroller of the Currency, SR Letter 11-7 Attachment, April (2011)

18. Föllmer, H., Schied, A.: Convex measures of risk and trading constraints. Financ. Stochast. 6(4), 429-447 (2002)

19. Föllmer, H., Schweizer, M.: Hedging of contingent claims under incomplete information. In: Davis, M., Elliott, R. (eds.) Applied Stochastic Analysis, Stochastics Monographs, vol. 5, pp. 389-414. Gordon and Breach, London (1991)

20. Föllmer, H., Sondermann, D.: Hedging of non-redundant contingent claims. In: Hildenbrand, W., MasCollel, A. (eds.) Contributions to Mathematicla Economics, pp. 205-223. NorthHolland, Amsterdam (1986)

21. Frittelli, M., Gianin, E.R.: Putting order in risk measures. J. Bank. Financ. 26(7), 1473-1486 (2002)

22. Jeanblanc, M., Yor, M., Chesney, M.: Mathematical Methods for Financial Markets. Springer, Berlin (2009)

23. Knight, F.H.: Risk, Uncertainty and Profit. Houghton Mifflin, Boston (1921)

24. Melino, A., Turnbull, S.M.: Misspecification and the pricing and hedging of long-term foreign currency options. J. Int. Money Financ. 14(3), 373-393 (1995)

25. Nalholm, M., Poulsen, R.: Static hedging and model risk for barrier options. J. Futures Mark. 26(5), 449-463 (2006)

26. Schoutens, W., Simons, E., Tistaert, J.: A perfect calibration! now what? Wilmott Mag. 2 , 66-78 (2004)

27. Schwartz, E.S., Smith, J.E.: Short-term variations and long-term dynamics in commodity prices. Manag. Sci. 46(7), 893-911 (2000)

28. Soner, M., Touzi, N., Zhang, J.: Quasi-sure stochastic analysis through aggregation. Electron. J. Probab. 16, 1844-1879 (2011). Article number 67 